\title{
Influence of daytime running lamps on visual reaction time of pedestrians when detecting turn indicators
}

By: Antonio Peña-García, Rocío de Oña, Antonio Espín, Fernando Aznar, Franscisco J. Calvo, Evaristo Molero and Juan de Oña

This document is a post-print versión (ie final draft post-refereeing) of the following paper:

Antonio Peña-García, Rocío de Oña, Antonio Espín, Fernando Aznar, Franscisco J.

Calvo, Evaristo Molero and Juan de Oña (2010) Influence of daytime running lamps on visual reaction time of pedestrians when detecting turn indicators. Journal of Safety Research, 41, 385-389.

Direct access to the published version: http://dx.doi.org/10.1016/j.jsr.2010.03.007 


\title{
Influence of Daytime Running Lamps on Visual Reaction Time of pedestrians when detecting Turn Indicators
}

\author{
A. Peña García, R. de Oña, A. Espín, F. Aznar, F.J. Calvo, E. Molero and J. de \\ Oña. \\ Departamento de Ingeniería Civil, Universidad de Granada, 18071 Granada, Spain \\ E-mail:pgarcia@ugr.es
}

\begin{abstract}
Introduction: This article describes one experiment that studied Daytime Running Lamps (DRL) influence on pedestrian detection of Turn Indicators. Method: An experimental device including one DRL and one Turn Indicator has been used in order to determine Visual Reaction Times (VRT) of 148 observers in different situations involving Turn Indicator activation. Such situations were combinations of three main variables: colour of DRL, separation between DRL and Turn Indicator and observation angle. Results: Significative changes in VRT were found depending on the configarations above, especially the observation angle and the colour of DRL. This second result definitively demonstrates that amber DRLs difficult Turn Indicators detection. Impact on industry: One of the main targets of this paper is to recommend carmakers to introduce only white DRLs on new vehicles. We also pretend to advise regulatory bodies working on automotive regulation about the consequences of allowing amber DRLs and also about the danger of introducing constrains on the distance between DRL and Turn Indicator without further experimental evidences.
\end{abstract}

Keywords: Headlamp, Daytime Running Lamp, Turn Indicator, Visual Reaction Time. 


\section{Introduction}

One of the most controversial functions when dealing with vehicle lighting and signalling and their influence on road safety is the Daytime Running Lamp (DRL) (Lachenmayr, 2003), whose main utility is to warn other users of the road about the presence and size of incoming vehicles during daytime. After many years of experience in several countries, DRLs are proved to be effective to avoid many traffic accidents (Theeuwes \& Riemersma, 1995; Williams \& Lancaster, 1995; Farmer \& Williams, 2002; Elvik, 1996; Elvik, 1993; Koornstra, Bijleveld \& Hagenzieker, 1997; Tofflemire \& Whitehead, 1997) but a big controversy arises when studying their visual interaction with other functions in automotive lighting like Turn Indicators (Palmer \& Kantowitz, 1994; Sivak, Flannagan, Schoettle \& Nakata, 2001; Regulation ECE 6, 2008).

Although the signalling functions like DRL and Turn Indicators have similar finalities (make vehicles detectable), not specialized people have rather different overviews of each one. While Turn Indicators and their purpose are well known functions, not everyone, especially in Europe, knows what a DRL is and what it is for. Hence, before any consideration, it is necessary to give a precise definition of DRL.

In countries where ECE Regulations are accepted (mainly in Europe), specific DRLs and their installation in motor vehicles are regulated by Regulation ECE 87 (2008) and Regulation ECE 48 (2008) respectively. According to these Regulations, "Daytime running lamp means a lamp facing in a forward direction used to make the vehicle more easily visible when driving during daytime". Since paragraph 2.7.25 of Regulation ECE 48 states that "National requirements may permit the use of other devices to meet this 
function", it is necessary to distinguish between "R87 DRL or specific DRL" and "other means" of signalling during daytime like Low Beam, dimmed High Beam or steady burning Turn Indicators.

DRLs in North America (USA and Canada) are regulated by Federal Motor Vehicle Safety Standard 108 (FMVSS 108, 2007), Canadian Motor Vehicle Safety Standard 108 (CMVSS 108, 2005) and SAE J2087 (1994). This standard defines DRL as a "Steady operating light function that is used to improve the conspicuity of a vehicle from the front and front-sides when the regular headlamps are not required for driving". In addition, the standard states that a "DRL function can be achieved with low or high beam headlamps, parking lamps, turn signal lamps, fog lamps, as well as dedicated lamps".

As shown in the paragraphs above, the main regulatory bodies define specific DRLs but also a vast variety of "other means of signalling during daytime". Anyhow, given the current convergence towards the use of specific DRLs, that will become mandatory in ECE countries in February 2011 (European Commission Directive 2008/89/EC, 2008), they are the target of this study. Their entry into force and subsequent increase on the roads in the next future is a challenging "leit motive" to look for answers to questions that maybe are not well understood yet.

\subsection{Daytime Running Lamps: different philosophies}

Regulation ECE 87 prescribes a photometric grid quite similar to those of the classic signalling functions in automotive lighting, whereas FMVSS 108 just requests one photometric value on the axis of the function. This requirement of FMVSS 108 is only 
for specific DRLs (the target of this paper) since other means of signalling during daytime have different photometric requirements. Here arises an important difference in the way to understand DRL between ECE countries and USA: whereas the first have well defined photometric requirements only for specific DRLs (the only ones to be used in the next future), in the United Stated there are clear photometric requirements even for alternative ways of lighting during daytime. This difference makes sense since it is not foreseen to demand specific DRLs by law there.

Another interesting remark is that Regulation ECE 48 does not impose any photometric constraint to either DRL or Turn Indicator whatever their mutual distance so far. However, FMVSS 108 requires that, for a DRL not optically combined with a Turn Indicator, the distance between its illuminating surface and the centre of the nearest Turn Indicator will be greater than $100 \mathrm{~mm}$ unless:

(i) The luminous intensity of the DRL is less than $2600 \mathrm{~cd}$ in the whole beam

(ii) The DRL is automatically switched off when the Turn Indicator is working.

This photometric constraint concerning the distance between both functions, that does not exist in ECE Regulation so far, will be one of the main targets of the experiment.

Finally, beyond these geometric and photometric remarks, there is another difference between prescriptions for DRL in USA and Europe, which will be also evaluated in this work: the colour of the light emitted by one DRL must be white in ECE countries whereas amber DRLs are permitted in USA and Canada according to SAE J578 (2006).

\subsection{Visual Reaction Time}


Given the dramatic influence of correct perception of vehicles by pedestrians and cyclists (Koornstra, Bijleveld \& Hagenzieker, 1997), a deep study of how this perception takes place is fully justified. The parameter used to quantify such perception has been Visual Reaction Time (VRT).

VRT can be defined as the interval of time between the application of a visual stimulus and the detection of a response from one observer (Luce, 1986). One of the best established properties of VRT is its inverse dependence on variations in luminance during detection tasks (Pins \& Bonet, 1996; Pins \& Bonet, 1997; Burr \& Corsale, 2001). This relationship, known as Piéron's law, is extremely important when considering the way to warn other users of the road about the presence of vehicles. Nevertheless, influence of near lights on reaction times of observers does not depend only on the luminance but also on the distance between such light sources. Observational experiments involving many observers are not easy to perform in automotive lighting (strong influence of environmental conditions, availability of observers ...), but the last advances, especially during daytime, require a deep revision of several concepts that were fully accepted in the past. Hence, one field experiment involving VRT could be extremely useful in order to clarify the actual interaction between turn indicators and DRLs as proved in this paper.

Furthermore, besides any theoretical model, the authors feel that the data provided by a field experiment can be extremely valuable in order to make situations as real as possible.

\section{Materials and Method}


In this work, we describe one experiment with 148 observers that has been carried out in order to measure their Visual Reaction Time in several situations involving DRL and Turn Indicator. The target of this experiment is to obtain some conclusions from their reaction time in the presence of a Turn Indicator that is suddenly and randomly switched on at different distances from a DRL.

With the results obtained it is expected to infer whether, as predicted by FMVSS 108, the distance between DRL and Turn Indicator has influence on the perception of this last. The experiment also pretends to identify which colour of DRL (white or amber) makes the perception of the Turn Indicator easier for pedestrians. This second question is very interesting from the regulatory point of view since, as mentioned above, DRLs can be either white or amber in the United States of America while they can be only white in the ECE countries.

For this last reason, and besides the measurements of reaction times, the participants in the experiment answered to the following subjective question about the colour of DRL: Do you think that amber DRLs difficult Turn Indicator detection?

\subsection{Experimental Device}

One headlamp with DRL was set on a stand that reproduces the conditions in the vehicle at a mounting height of $900 \mathrm{~mm}$ on the ground. Low and high beam remained switched off all the time. The module hosting the Turn Indicator, which was independent from the main headlamp, was fitted to the same stand and placed under the headlamp. It could be sliced up and down through a bar that allowed its mounting height to change with no angular deviation of its optical axis. 


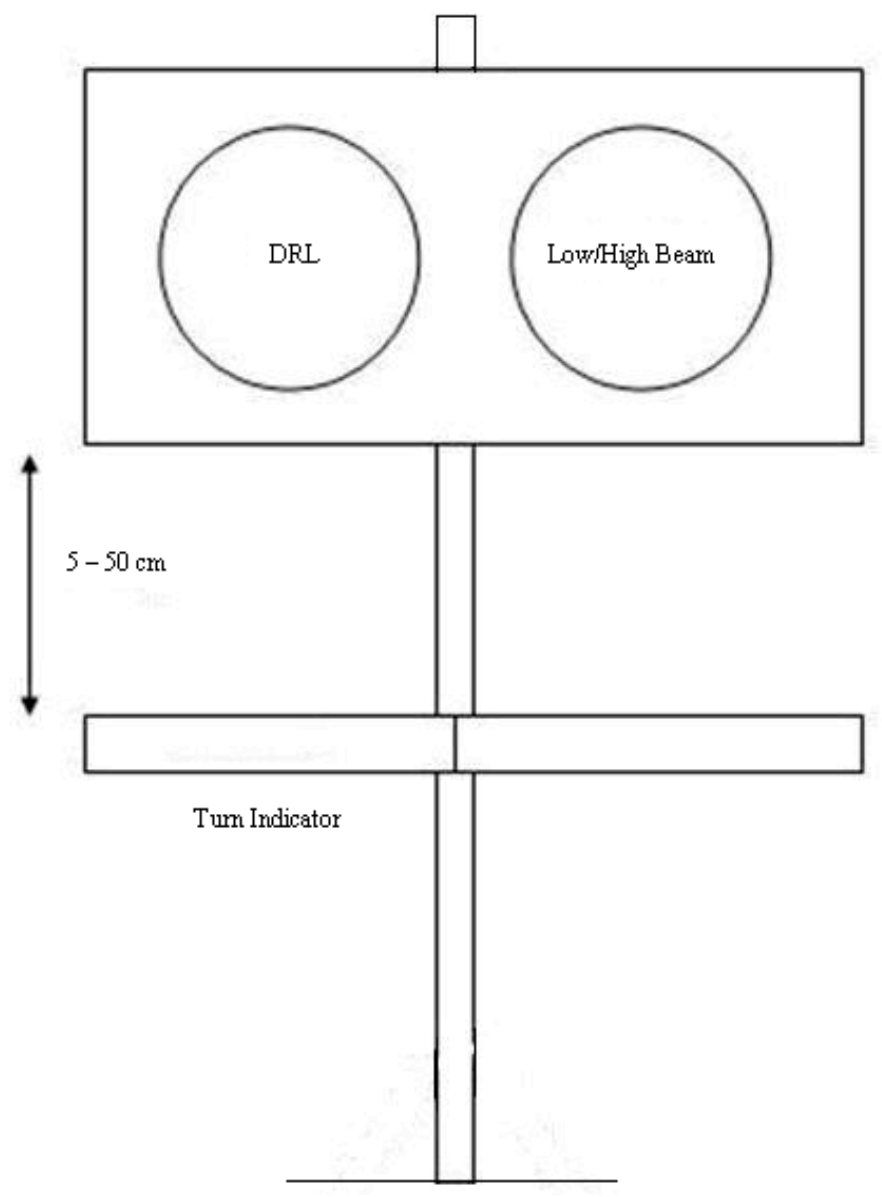

Figure 1. Scheme of the set headlamp - signalling module on sliding fixture

The DRL used a P21W bulb when it was white and a PY21W when amber, both powered at $13.5 \mathrm{~V}$. For the Turn Indicator, a H21W bulb was used at the same voltage. All these bulbs are approved according to Regulation ECE 37 (2008).

In order to accurately distribute the light, the DRL is performed by a concave reflector of circular shape made of small mirrors that direct the beams. The reflector of Turn Indicator is similar to but its shape is rectangular and it includes a piece of polycarbonate with optical grooves (fresnel) to help re-direction of light by reflection. Inside the module there is a Position Lamp (bulb and reflector) which remained off during the whole experiment. 


\subsection{Participants}

For this experiment, 148 volunteers (50 women and 98 men), have been recruited among the students of Lighting Technology of Civil Engineering (University of Granada, Spain). The mean age was 20,0 years with extreme values of 18 and 26 years and a standard deviation of 1,37 . The mean height was $1,75 \mathrm{~m}$ with extreme values of 1,52 and $1,91 \mathrm{~m}$ and standard deviation of 0,09 .

\subsection{Method}

The experiment was carried out in a closed street during daytime in a very sunny day in order to reproduce the most unfavourable conditions for pedestrian detection of vehicles. In all cases, the distance between participants and experimental device was $25 \mathrm{~m}$, which is the standard distance of measurement required by the ECE Regulations.

In each measurement, participants settled their eyes on one object situated behind the experimental device but slightly higher while the DRL in the experimental device was continuously lit. Under these conditions, participants had to detect the activation of the Turn Indicator, which was remotely switched on after a random time of wait and their VRT were measured. Such measurements were carried out in several situations as summarized in Table 1.

\begin{tabular}{|c|c|c|c|}
\hline Colour of DRL & $\begin{array}{c}\text { DRL-Turn Signal } \\
\text { distance } \mathbf{( c m )}\end{array}$ & $\begin{array}{c}\text { Angle observer- } \\
\text { device }\end{array}$ & Notation \\
\hline Amber & 5 & $0^{\circ}$ & A-5-0 \\
\hline Amber & 50 & $0^{\circ}$ & A-50-0 \\
\hline Amber & 5 & $20^{\circ}$ & A-5-20 \\
\hline
\end{tabular}




\begin{tabular}{|c|c|c|c|}
\hline Amber & 50 & $20^{\circ}$ & A-50-20 \\
\hline White & 5 & $0^{\circ}$ & W-5-0 \\
\hline White & 50 & $0^{\circ}$ & W-50-0 \\
\hline White & 5 & $20^{\circ}$ & W-5-20 \\
\hline White & 50 & $20^{\circ}$ & W-50-20 \\
\hline
\end{tabular}

Table 1. Summary of measures

The target of measuring VRT with amber and white DRLs, was to determine the most convenient colour of DRL to avoid visual masking of Turn Indicators.

The target of measuring VRT with different distances between Turn Indicator and DRL, was to look for favourable or unfavourable evidences that might recommend regulatory bodies to request more intense Turn Indicators when located near from DRLs.

The target of measuring VRT with different angular positions between observer and experimental device, was to determine the lack of visual performance in peripheral vision for detection tasks.

\subsection{Statistical methods}

The statistical analysis of the results was performed by means of analysis of variance (ANOVA), on a quantitative dependent variable and independent variables (factors). Analysis of variance is used to test the hypothesis that several means are not the same. In our analyses we performed several two- and three-factor ANOVA for different response variables. In addition to determining that differences between the means exist, several post-hoc LSD tests were considered on factor levels. Interactions between factors were considered, in order to determine if the presence/absence of a factor level increases/decreases the effect on the response variable. Study of Residuals and Bartlett 
tests were performed for checking assumptions of normality and homoscedasticity, respectively (Abraira \& Perez, 1996; Myers et al., 2002). Calculations were performed using R-statistical program (R Development Core Team, 2006).

\section{Results}

After some training and control measures for the participants to become familiar with the situation, VRT were measured with the results presented in Tables 2 and 3.

\begin{tabular}{|l|c|c|c|c|}
\hline & A-5-0 & A-50-0 & W-5-0 & W-50-0 \\
\hline Average value & 0,98 & 0,95 & 0,93 & 0,97 \\
\hline Standard deviation & 0,25 & 0,14 & 0,13 & 0,18 \\
\hline Max. Value & 3,00 & 1,66 & 1,45 & 2,00 \\
\hline Min. Value & 0,75 & 0,72 & 0,75 & 0,66 \\
\hline Valid cases & 148 & 148 & 148 & 148 \\
\hline
\end{tabular}

Table 2. Results obtained for front observation. Figures in seconds.

\begin{tabular}{|l|c|c|c|c|}
\hline & $\mathbf{A - 5 - 2 0}$ & $\mathbf{A - 5 0 - 2 0}$ & $\mathbf{W - 5 - 2 0}$ & $\mathbf{W - 5 0 - 2 0}$ \\
\hline Average value & 1,04 & 1,05 & 0,99 & 0,95 \\
\hline Standard deviation & 0,34 & 0,86 & 0,27 & 0,14 \\
\hline Max. value & 3,53 & 10,90 & 3,47 & 1,56 \\
\hline Min. value & 0,72 & 0,75 & 0,69 & 0,75 \\
\hline Valid cases & 147 & 148 & 148 & 148 \\
\hline
\end{tabular}

Table 3. Results obtained for observation at $20^{\circ}$. Figures in seconds.

A three-way ANOVA (factors colour-separation-angle) was performed to analyze the visual reaction time by colour of DRL, separation between DRL and Turn Indicator and observation angle as factors. The results are summarized in Table 4. 


\begin{tabular}{|c|c|c|}
\hline & VRT (s) & Count \\
\hline Average & 0,982 & 1183 \\
\hline \multicolumn{3}{|l|}{ Colour of DRL } \\
\hline Amber & $1,003 \mathrm{a}$ & 591 \\
\hline White & $0,960 \mathrm{~b}$ & 592 \\
\hline \multicolumn{3}{|l|}{$\begin{array}{l}\text { Separation between DRL } \\
\text { and Turn Indicator }\end{array}$} \\
\hline $5 \mathrm{~cm}$ & 0,985 & 591 \\
\hline $50 \mathrm{~cm}$ & 0,978 & 592 \\
\hline \multicolumn{3}{|l|}{ Observation angle } \\
\hline $0^{\circ}$ & $0,955 \mathrm{a}$ & 592 \\
\hline $20^{\circ}$ & $1,008 \mathrm{~b}$ & 591 \\
\hline
\end{tabular}

Levels of the same factor without letter mean that the differences are statistically non-significant. a, b: denotes differences statistically significant $(\mathrm{p}<0.05)$

Table 4. Visual Reaction Time (VRT) vs. colour of DRL, separation between DRL and turn indicator and observation angle.

A carefully analysis of Table 4 shows the following:

- The LSD test shows that the colour of DRL has a statistically significant $(p<0.05)$ effect on the visual reaction time. The VRT for white DRL is lower ( 0.960 seconds in average) than the VRT for amber DRL (1.003 seconds).

- Also, the LSD test shows that the observation angle has a statistically significant $(p<0.05)$ effect on the visual reaction time. The VRT for front observation is lower $\left(0.955\right.$ seconds in average) than the VRT for observation at $20^{\circ}(1.008$ seconds).

- However, the same test shows that the separation between DRL and Turn Indicator does not have a statistically significant ( $>0.05)$ effect on VRT.

- Interaction analysis of principal factors does not identify statistical significance for the three two-factor interactions and for the three-factor interaction. 
After the experiment, participants were encouraged to discuss about the results and find possible influence of experimental conditions on the results obtained. Their opinions and feelings about the different configurations and situations have been extremely important for the researchers to obtain some of the conclusions summarized in the next section.

\section{Conclusions and recommendations}

Several immediate conclusions can be deduced from the measures in Tables 2 and 3 and its analysis (Table 4):

1.- When the colour of DRL is amber, average VRT is higher if compared with white DRL. It proves that, when both functions have the same colour, visual performance of Turn Indicators is diminished. But, besides the fact that lights with similar colours can lead to confusion when they are near, this result also agrees with some more theoretical experiments (Nissen, Pokorny \& Smith, 1979; Ueno, Pokorny \& Smith, 1985; Ueno \& Swanson, 1989; Martínez, Jiménez, Rubiño \& Jiménez, 1993), that showed higher VRTs for visual tasks in environments whose chromaticity was around $570 \mathrm{~nm}$ (near from amber).

This conclusion also agrees with the feelings of the participants after the experiment. Thus, when asked whether, to their understanding, amber DRLs difficult Turn Indicator detection, $70 \%$ of participants answered that amber DRLs make detection of Turn Indicators more difficult. 
2.- For observation angles of $20^{\circ}$, average VRT is higher than for observation angles of $0^{\circ}$ (frontal observation). This result, which is logical if we consider that visual performance is much higher in foveal vision, that is, observation angles $<10^{\circ}$ (Romero, García \& García, 1996), must be seriously considered because most knoks downs to pedestrian and cyclists take place under notable angles between directions of cars and victims.

3.- Separation between DRL and Turn Indicator has not been proved to be statistically significant according to the ANOVA on the results obtained. This conclusion is extremely important and demonstrates that photometric performance of signalling functions does not imperatively decrease when they are close. Even, the lowest average VRT arises from the configuration Turn Signal - white DRL when both functions are near $(5 \mathrm{~cm})$.

This conclusion strongly suggests that the recent proposals of amendment to Regulation ECE 6 (2008) (Turn indicators for motor vehicles) to request more intense Turn Indicators when they are near from DRL, should be more carefully studied.

Conclusions above, demonstrate that white DRLs are much more efectively discriminated from Turn Indicators. Thus, given that the vast majority of cars incorporate Turn Indicators and DRLs in the same lamp (that is, they are quite near), we suggest that regulations allowing amber DRLs should deeply consider the effects on their interaction with Turn Indicators. 
The evidences found also encourage us to suggest that constrains on mutual distance between both functions must be deeply studied before being introduced in Regulations. In summary, the positive experience and the logic of the results obtained, evidence the necessity of carrying out more experiments on the mutual interaction between different automotive lights in order to harmonize the different regulations with the target of improving safety on the road.

\section{Acknowledgments}

We specially thank the students that participated in this experiment for their intelligent remarks and implication. Without their collaboration this experiment would have never been possible.

\section{References}

Abraira, V. \& Pérez, A. (1996). Métodos Multivariantes en Bioestadística. Centro de Estudios Ramón Areces. Madrid, Spain.

Burr, D.C., \& Corsale, B. (2001), Dependence of reaction times to motion onset on luminance and chromatic contrast. Vision Research, 41, 1039-1048.

Elvik, R. (1996). A meta-analysis of studies concerning the safety effects of daytime running lights on cars. Accident Analysis and Prevention, 28 (6): 685-694.

Elvik, R. (1993). The effects on accidents of compulsory use of daytime running lights for cars in Norway. Accident Analysis and Prevention, 25(4), 383-398.

European Commission Directive 2008/89/EC (2008).

Farmer, C. M., \& Williams, A. F. (2002). Effects of daytime running lights on multiplevehicle daylight crashes in the United States. Accident Analysis and Prevention, 34 (2): 197-203. 
Koornstra, M., Bijleveld, F. \& Hagenzieker, M. (1997). The safety effects of daytime running lamps. Research (No. R-97-36), SWOV Institute for Road Safety, Leidschendam, The Netherlands.

Lachenmayr, B. (2003). Daytime running light (DRL). Pro and contra. Ophthalmologe 100 (10): 836-842.

Luce, R. D. (1986). Response times. Oxford University press, New York.

Martínez, P., Jiménez, J. R., Rubiño, M. \& Jiménez, L. (1993). Color and visual reaction Time. Die Farbe, 39, 1-6.

Myers, R. H., Montgomery, D.C. \& Vining, G. G. (2002). Generalized Linear Models, With Applications in Engineering and the Sciences. John Wiley \& Sons, Inc., New York.

National Highway Traffic Safety Administration (NHTSA). (2007). Federal Vehicle Motor Safety Standard 108.

Nissen, M.J., Pokorny, J. \& Smith, V. C. (1979). Chromatic information processing. J. Exp. Psycho, 5, 405-419.

Palmer, J. C., \& Kantowitz, B. (1994). Daytime running lights and turn signal masking. Report No. DOT HS 808 221, National Highway Traffic Safety Administration, Washington, D.C. USA.

Pins, D. \& Bonnet, C. (1996). On the relation between stimulus intensity and processing time: Pieron's law and choice reaction time. Perception \& Psychophysics, 58, 390-400. Pins, D. \& Bonnet, C. (1997). Reaction times reveal the contribution of the different receptor components in luminance perception. Psychonomic Bulletin \& Review, 4, 359366. 
R Development Core Team (2006). R: A language and environment for statistical computing. R Foundation for Statistical Computing, Vienna, Austria. http://www.Rproject.org.

Romero, J., García, J. A. \& García, A. (1996). Curso introductorio a la Óptica Fisiológica. Comares, Granada, Spain.

Sivak, M., Flannagan, M. J., Schoettle, B., \& Nakata, Y. (2001). Masking of front turn signals by headlamps in combination with other front lamps. Lighting Research and Technology, 33: 233-241.

Society of Automotive Engineers, Inc. (SAE). (2006). SAE J578 DEC06.

Society of Automotive Engineers, Inc. (SAE). (1994). SAE J2087 AUG91.

Theeuwes, J., \& Riemersma, J. (1995). Daytime running lights as a vehicle collision countermeasure - The Swedish evidence reconsidered. Accident Analysis and Prevention, 27 (5): 633-642.

Tofflemire, T. C. \& Whitehead, P. C. (1997). An Evaluation of the Impact of Daytime Running Lights on Traffic Safety in Canada. J. Saf. Res., 28(4), 257-272.

Transport Canada - Road Safety and Motor Vehicle Regulation Directorate. (2005). Canadian Vehicle Motor Safety Standard 108.

Ueno, T., Pokorny, J. \& Smith, V. C. (1985). Reaction times to chromatic stimuli. Vision res, 25, 1623-1627.

Ueno, T., \& Swanson, W. H. (1989). Response pooling between chromatic and luminance systems. Vision Res, 29, 325-333.

United Nations Economic Commission for Europe (UNECE). (2008). Regulation ECE No. 6 - Uniform provisions concerning the approval of vehicles with regard to the installation of lighting and light-signalling devices. 
United Nations Economic Commission for Europe (UNECE). (2008). Regulation ECE No. 37 - Uniform provisions concerning the approval of filament lamps for use in approved lamps units on power-driven vehicles and of their trailers.

United Nations Economic Commission for Europe (UNECE). (2008). Regulation ECE No. 48 - Uniform provisions concerning the approval of vehicles with regard to the installation of lighting and light-signalling devices.

United Nations Economic Commission for Europe (UNECE). (2008). Regulation ECE No. 87 - Uniform provisions concerning the approval of daytime running lamps for power-driven vehicles.

Williams, A. F. \& Lancaster, K. A. (1995). The prospects of daytime running lights for reducing vehicle crashes in the United-States. Public Health Reports, 110(3), 233-239. 